\title{
When is Big Data big enough? Implications of using GPS-based surveys for travel demand analysis
}

Akshay Vij (corresponding author)

Senior Research Fellow

Institute for Choice

University of South Australia

Level 13, 140 Arthur Street

North Sydney, NSW 2060

vij.akshay@gmail.com

+61(08) 83020817

K. Shankari

Ph.D. Candidate

Department of Electrical Engineering and Computer Sciences

University of California, Berkeley

465 Soda Hall

Berkeley, CA 94720-1776

shankari@eecs.berkeley.edu

$+1(650) 454-6036$ 


\begin{abstract}
A number of studies in the last decade have argued that Global Positioning Systems (GPS) based survey offer the potential to replace traditional travel diary surveys. GPS-based surveys impose lower respondent burden, offer greater spatiotemporal precision and incur fewer monetary costs. However, GPS-based surveys do not collect certain key inputs required for the estimation of travel demand models, such as the travel mode(s) taken or the trip purpose, relying instead on data-processing procedures to infer this information. This study assesses the impact that errors in inference can have on travel demand models estimated using data from GPS-based surveys and proposes ways in which these errors can be controlled for during both data collection and model estimation. We use simulated datasets to compare performance across different sample sizes, inference accuracies, model complexities and estimation methods. Findings from the simulated datasets are corroborated with real data collected from individuals living in the San Francisco Bay Area, United States. Results indicate that the benefits of using GPS-based surveys will vary significantly, depending upon the sample size of the data, the accuracy of the inference algorithm and the desired complexity of the travel demand model specification. In many cases, gains in the volume of data that can potentially be retrieved using GPS devices are found to be offset by the loss in quality caused by inaccuracies in inference. This study makes the argument that passively collected GPS-based surveys may never entirely replace surveys that require active interaction with study participants.
\end{abstract}


Keywords: GPS-based surveys, travel diary surveys, travel demand models, inference error 


\section{List of Figures}

Figure 1: Example confusion matrix, adapted from NCHRP Report 775 (Wolf et al. 2014), for a neural network travel mode inference algorithm

Figure 2: General form of the confusion matrix used in experiments 1 and 2

Figure 3: A boxplot showing the median, 25th and 75th percentile, and minimum and maximum value of travel time $(\$ / \mathrm{hr})$ for experiment 1 as estimated using the unweighted likelihood function

Figure 4: A boxplot showing the median, 25th and 75th percentile, and minimum and maximum value of travel time $(\$ / \mathrm{hr})$ for experiment 1 as estimated using the weighted likelihood function

Figure 5: A boxplot showing the median, 25th and 75th percentile, and minimum and maximum value of in-vehicle travel time $(\$ / \mathrm{hr})$, value of walking time $(\$ / \mathrm{hr})$, value of biking time $(\$ / \mathrm{hr})$ and value of waiting time ( $\$ / \mathrm{hr})$ for experiment 2 , as estimated using the unweighted likelihood function

Figure 6: A boxplot showing the median, 25th and 75th percentile, and minimum and maximum value of in-vehicle travel time $(\$ / \mathrm{hr})$, value of walking time $(\$ / \mathrm{hr})$, value of biking time $(\$ / \mathrm{hr})$ and value of waiting time $(\$ / \mathrm{hr})$ for experiment 2 , as estimated using the weighted likelihood function

Figure 7: General form of the confusion matrix used in experiment 3

Figure 8: A boxplot showing the median, 25th and 75th percentile, and minimum and maximum value of in-vehicle travel time $(\$ / \mathrm{hr})$, value of walking time $(\$ / \mathrm{hr})$, value of biking time $(\$ / \mathrm{hr})$ and value of waiting time $(\$ / \mathrm{hr})$ for experiment 3, as estimated using the unweighted likelihood function

Figure 9: A boxplot showing the median, 25th and 75th percentile, and minimum and maximum value of in-vehicle travel time $(\$ / \mathrm{hr})$, value of walking time $(\$ / \mathrm{hr})$, value of biking time $(\$ / \mathrm{hr})$ and value of waiting time $(\$ / \mathrm{hr})$ for experiment 3, as estimated using the weighted likelihood function 
Figure 10: A plot comparing the value of time $(\$ / \mathrm{hr})$ as estimated by maximizing the unweighted and weighted likelihood functions given by equations (11) and (12), respectively, as a function of the average accuracy of the inference algorithm

Figure 11: A bar and line plot where the bar plot represents the value of time ( $\$ / \mathrm{hr})$ estimated by maximizing the weighted likelihood function given by equation (5) and the inferred data is supplemented with ground truth, and the line plot represents the percentage of trips for which the accuracy of the inference algorithm is below $90 \%$ and ground truth is used, plotted as a function of the average accuracy of the inference algorithm 


\section{List of Tables}

Table 1: Distributions for travel mode level-of-service attributes for experiment 1

Table 2: Parameter values for experiment 1

Table 3: Distributions for travel mode level-of-service attributes for experiments 2 and 3

Table 4: Parameter values for experiments 2 and 3

Table 5: Elements of the confusion matrix used in experiment 3 as a function of the average accuracy of the inference algorithm 


\section{Introduction}

Traditional models of individual and household travel and activity behavior are estimated using travel diary datasets that ask a small subset of the population of interest to record over a period of one or two days which activities were conducted where, when, for how long, with whom and using what mode of travel. For example, the New York Best Practices Model (NYBPM), the activity-based model of travel demand developed for the New York Metropolitan Region, was estimated using travel diary data from about 28,000 individuals collected over a period of one day (Parsons Brinkerhoff Quade \& Douglas, Inc. 2005). The size of that and other similar travel diary datasets pales in comparison to the volume of information that can potentially be retrieved from new technologies, such as Global Positioning System (GPS) sensors and smartphones, and social media platforms, such as Twitter and Facebook, both now and in the future.

Advances in GPS technologies in particular have received substantive attention in the last decade (for an exhaustive review of the literature, the reader is referred to Zmud et al. 2013; Shen \& Stopher 2014; and Wolf et al. 2014). Early applications sought to supplement extant methods of travel diary data collection that rely on self-reporting, such as mail-back, phone-based or door-to-door travel diary surveys, through their ability to control for factors such as trip underreporting (Pierce et al. 2003; Wolf et al. 2003; Zmud \& Wolf 2003; Bricka \& Bhat 2007; Bachman et al. 2012). A number of regional household travel surveys have since adopted GPS-based surveys as a subcomponent to improve the accuracy and completeness of data collected using these more traditional methods. Recent examples include the Atlanta Regional Travel Survey, conducted in 2011, and the California Household Travel Survey, conducted in 2012 and 2013.

However, the long-term objective has always been the development of GPS-based surveys that can collect all the information that is usually collected by traditional travel diary surveys, but with very little input from survey participants. Thus far, there have been three large-scale travel surveys that have relied entirely on GPS sensors for the collection of travel diary data. The Ohio Department of Transportation 
initiated the first exclusively GPS household travel survey, conducted in the Cincinnati metropolitan area over 2009 and 2010 with a sample of 2,583 households (Stopher et al. 2012), and followed it with the Northeast Ohio Regional Travel Survey conducted over 2012 and 2013 in the Cleveland metropolitan area with a sample of 4,545 households. The regional planning agency in Jerusalem administered a similar exclusively GPS household travel survey over 2010 and 2011 with a sample of 8,800 households. As the technology matures, GPS-based surveys are expected eventually to replace traditional travel diary surveys (Wolf et al. 2001; Stopher et al. 2008).

The advantages of using GPS-based surveys are manifold. They impose fewer requirements on survey respondents, offer greater spatiotemporal precision and are potentially cheaper to implement. Nevertheless, as pointed out by Shen \& Stopher (2014), GPS-based surveys "cannot record travel mode, trip purpose or the number of occupants in a private vehicle - all important attributes in a traditional travel survey. Therefore, data-processing procedures become critical to the usefulness of GPS surveys, because there would be insufficient information for travel modelling purposes without the results of the processing." Existing GPS-based surveys ask a subset of the sample population to participate in prompted-recall surveys where the respondents are asked to confirm trip details. The data thus collected is used to infer the same trip details for the remainder of the sample population. Numerous algorithms have been proposed for inferring one or more of these missing details from the GPS data, augmented in many cases with additional sources, such as accelerometer readings from smartphones (e.g. Feng \& Timmermans 2013), land use characteristics from Geographic Information Systems (GIS) databases (e.g. Bohte \& Maat 2009) or 'check-ins' from social media applications (e.g. Hasan \& Ukkusuri 2014). However, even the most successful inference algorithm will have some error associated with it. Most published studies in the literature report average accuracies of $60-90 \%$. For example, the best practices travel mode inference model recommended by the National Cooperative Highway Research Program (NCHRP) Report 775, titled 'Applying GPS Data to Understand Travel Behavior,' has an average accuracy of $82 \%$ (Wolf et al. 2014). Errors in inference can potentially compromise the quality of data 
collected through GPS-based surveys and the validity of travel demand models developed using this data. And yet, to the best of our knowledge, no study has systematically examined the implications of using low-quality big data for traditional modes of analyses.

The objectives of this study are two-fold: (1) to evaluate the impact of errors in inference on travel demand models estimated with GPS data using simulated and real datasets; and (2) to develop ways in which these errors can be controlled for through data collection methods and estimation procedures. To be clear, we do not wish to compare the performance of different inference algorithms. There are numerous studies in the literature that have already done so (refer to Shen \& Stopher 2014 or Wolf et al. 2014 for a comprehensive list). Rather, our objective is to determine, for a given inference algorithm with a measured level of accuracy and precision that is used to impute missing information from GPS-based surveys, the validity of travel demand models estimated using this imputed data.

The paper is structured as follows: Section 2 describes a series of Monte Carlo experiments that compare model performance across different sample sizes, inference accuracies, model complexities and estimation methods; Section 3 uses actual GPS data collected from individuals residing in the San Francisco Bay Area, United States to corroborate findings from the Monte Carlo experiments; and Section 4 concludes the paper with a summary of findings and implications.

\section{Monte Carlo Experiment}

In this section, we simulate a series of three Monte Carlo experiments to assess the impact of inference errors on estimation results as a function of the accuracy of the inference algorithm, the complexity of the desired travel demand model specification, the sample size of the observed data and the estimation method used to recover parameter estimates. A Monte Carlo experiment is especially useful because the true parameters underlying the data generating process are known, and the impact of inference errors can 
be evaluated under a wide variety of conditions, leading to more generalizable results that aren't specific to any one dataset.

Each of the three Monte Carlo experiments comprises three steps. First, we simulate datasets for a hypothetical Random Utility Maximization (RUM) model of travel mode choice behavior. The RUM model is by far the most popular model among studies on individual and household travel and activity behavior, and travel mode choice behavior perhaps the most widely studied problem (see, for example, Ben-Akiva \& Lerman 1985). Second, we simulate the probability that the chosen travel mode, as predicted by the RUM model, is correctly identified by a hypothetical travel mode inference algorithm, using confusion matrices for travel mode inference algorithms commonly observed in the literature. And third, we recover the parameter estimates for the model of travel mode choice behavior through two different estimation methods, using the explanatory variables simulated in step 1 and the inferred choices simulated in step 2, and compare these estimates with their true values. The model specification and confusion matrices are varied across experiments. Over the course of the subsequent paragraphs, we describe each of the three steps in greater general detail. Sections 2.1, 2.2 and 2.3 provide additional information on the specifics of the model specification and confusion matrices employed as part of each of the three experiments, and how these differences influenced the estimation results. Though the example used here evaluates the impact of errors in travel mode inference on estimation results for a model of travel mode choice behavior, the framework of the Monte Carlo experiment is general in that it can be easily adapted to evaluate the impact of other sources of measurement error, as might result from trip underreporting, or from inference errors with regards to other trip characteristics, on estimation results for models of behavior in other contexts.

Some of the earliest applications of the RUM model have been in the field of travel and activity behavior, with travel mode choice behavior attracting particular attention (see, for example, McFadden 2001). How individuals interact with the urban transportation system holds important implications for the health of our cities and the global environment at large. A greater comprehension of the many factors that shape 
travel mode choice behavior is essential to the successful design of systems that serve the immediate needs of the population while satisfying long-term societal objectives. Given the importance of the subject, we build a hypothetical trip-based model of travel mode choice behavior for our experiment. Our model formulation is loosely based on travel mode choice model components estimated as part of larger activity-based travel demand model frameworks. However, our model formulation is more parsimonious than most travel mode choice models in practice to allow us to explore the impact of inference accuracies on the estimates for each of the model parameters in greater depth than would be possible with a more complex specification containing a larger number of total parameters.

We assume that for a given trip, a decision-maker can choose between four travel modes: walk, bike, car and transit. Let $U_{n j}$ be the utility of travel mode $\mathrm{j}$ as perceived by decision-maker $\mathrm{n}$, defined as follows:

$$
U_{n j}=V_{n j}+\varepsilon_{n j}=\boldsymbol{\beta}^{\prime} \mathbf{X}_{n j}+\varepsilon_{n j}
$$

, where $V_{n j}$ is the systematic component of the utility and $\varepsilon_{n j}$ is the stochastic component, assumed to be i.i.d. Gumbel with location zero and scale parameter one. The systematic component is defined as a linear function of the vector of explanatory variables $\mathbf{X}_{\mathbf{n j}}$ and the vector of model parameters $\boldsymbol{\beta}$, denoting sensitivity to each of these explanatory variables. Bolded model parameters and variables denote vectors, unbolded model parameters and variables denote scalars. The vector of explanatory variables may consist of alternative-specific variables, such as travel times and costs, individual-specific variables, such as gender and income, and interactions between these two sets of variables. Decision-makers are assumed to be utility-maximizing in that they choose that travel mode that offers them the greatest utility:

$$
y_{n j}=\left\{\begin{array}{l}
1 ; \text { if } U_{n j} \geq U_{n j} \text { for } j^{\prime}=1, \ldots, J \\
0 ; \text { otherwise }
\end{array}\right.
$$


, where $y_{n j}$ is an indicator of the actual choice. The assumption that $\varepsilon_{n j}$ is i.i.d. Gumbel with location zero and scale one results in the familiar multinomial logit expression for the probability that decisionmaker $\mathrm{n}$ chooses travel mode $\mathrm{j}$ :

$$
\mathrm{P}\left(\mathrm{y}_{\mathrm{nj}}=1 \mid \boldsymbol{\beta} ; \mathbf{X}_{\mathbf{n}}\right)=\frac{\exp \left(\boldsymbol{\beta}^{\prime} \mathbf{X}_{\mathbf{n} \mathbf{j}}\right)}{\sum_{\mathbf{j}^{\prime}} \exp \left(\boldsymbol{\beta}^{\prime} \mathbf{X}_{\mathbf{n j}^{\prime}}\right)}
$$

, where $\mathbf{X}_{\mathbf{n}}$ is a matrix whose $\mathrm{j}^{\text {th }}$ column is the vector $\mathbf{X}_{\mathbf{n j}}$. Following the methodology proposed by Williams \& Ortúzar (1982) and the approach outlined by Raveau et al. (2010), the vector of explanatory variables $\mathbf{X}_{\mathbf{n j}}$ are synthesized using distributions that are reflective of values that would be observed in empirical data. The vector of model parameters $\boldsymbol{\beta}$ is chosen such that it satisfies three conditions. First, the marginal rates of substitution between the explanatory variables are consistent with values observed by studies in the literature to ensure that the experiment design is as realistic as possible. Second, the partworth utilities of each of the explanatory variables, as represented by the product between that variable and the corresponding parameter, are comparable in terms of magnitude. If this is not the case, one of the attributes could potentially dominate the utility function, and it may be hard to empirically isolate the effect of other variables. And finally, the scale of the model is set such that the error rate for the data is roughly $20 \%$, i.e. one in five simulated decision-makers change their choice because of the stochastic component, thereby ensuring that the decision-making process is neither completely deterministic nor completely stochastic ${ }^{1}$.

\footnotetext{
${ }^{1}$ This is achieved by adjusting the scale of the utility through corresponding adjustments in the model parameter values. The scale of the utility is fixed by constraining the scale parameter of $\varepsilon_{\mathrm{nj}}$, the stochastic component of the utility, to be one, or the variance of $\varepsilon_{\mathrm{nj}}$ to be $\pi^{2} / 6$. The smaller the magnitude of the model parameters, the smaller the magnitude of the systematic component of the utility in relation to the stochastic component, the greater the variance in the data, and the greater the error rate. For example, if we find that for a given vector of parameter values the error rate is higher than the desired value of $20 \%$, we multiply all parameter values by some factor greater than 1 ,
} 
Equations (1)-(3) provide a blueprint for generating any number of observations for a model of travel mode choice behavior with any desired level of complexity. However, for a given observation, the travel mode actually chosen, as identified by equation (3), will not always be the same as the travel mode inferred to have been chosen. A number of studies have explored how the chosen travel mode might be inferred from GPS data, augmented in many case with data from other sensors, such as accelerometers and magnetometers, and data from other sources, such as GIS databases and General Transit Feed Specification (GTFS) feeds, using classifiers such as conditional random fields, decision trees, support vector machines, artificial neural networks, etc. (see, for example, Gonzalez et al. 2008, Reddy et al. 2008, Zheng et al. 2010, Stenneth et al. 2011, Bolbol et al. 2012, Hemminki et al. 2012 and Carrel et al., 2015). As mentioned before, the objective of this study is not to compare different inference algorithms and their ability to recover missing data. Rather, our objective is to examine, for a given inference algorithm with a measured level of accuracy, used to impute missing information from GPS-based surveys, the validity of travel demand models estimated using this imputed data. In this section specifically, our objective is to simulate errors in travel mode inference using hypothetical confusion matrices.

\section{$<$ Insert Figure 1 roughly around here>}

Figure 1 shows an example confusion matrix, adapted from NCHRP Report 775, for a neural network inference algorithm, recommended by the report as a best practice travel mode inference algorithm (Wolf et al. 2014). Each cell in the matrix represents the probability of inferring that an individual chose a particular travel mode given the travel mode that was actually chosen. For example, the probability that the inferred travel mode is car given that the actual travel mode is walk is 0.06 . Again, to keep the

preserving the original marginal rate of substitution between variables, until the error rate is acceptably close to $20 \%$. And if the error rate is too low, we multiply all parameter values by some factor less than 1, until the error rate is acceptably close to $20 \%$. 
experiment design as realistic as possible, the confusion matrices are generated based on what has been reported in the literature.

To underline differences between actual and inferred choices, we introduce the variable $\mathrm{y}_{\mathrm{nj}}^{\prime}$, defined as follows: $\mathrm{y}_{\mathrm{nj}}^{\prime}$ equals one if individual $\mathrm{n}$ is inferred to choose travel mode $\mathrm{j}$, and zero otherwise. For each of the datasets simulated using the process outlined above, estimates for the model parameters may then be recovered by maximizing the following likelihood function:

$$
\begin{aligned}
\mathrm{L}\left(\boldsymbol{\beta} \mid \mathbf{y}^{\prime}, \mathbf{X}\right) & =\prod_{n} \mathrm{P}\left(\mathbf{y}_{\mathbf{n}}^{\prime} \mid \boldsymbol{\beta} ; \mathbf{X}_{\mathbf{n}}\right) \\
& =\prod_{n} \prod_{j}\left[\mathrm{P}\left(\mathrm{y}_{\mathrm{nj}}=1 \mid \boldsymbol{\beta} ; \mathbf{X}_{\mathbf{n}}\right)\right]^{\mathrm{y}_{\mathrm{nj}}^{\prime}} \\
& =\prod_{\mathbf{n}} \prod_{j}\left[\frac{\exp \left(\boldsymbol{\beta}^{\prime} \mathbf{X}_{\mathbf{n j}}\right)}{\sum_{\mathbf{j}^{\prime}} \exp \left(\boldsymbol{\beta}^{\prime} \mathbf{X}_{\mathbf{n} \mathbf{j}^{\prime}}\right)}\right]^{\mathrm{y}_{\mathrm{nj}}^{\prime}}
\end{aligned}
$$

, where $\mathbf{y}_{\mathbf{n}}^{\prime}$ is a vector whose $\mathrm{j}^{\text {th }}$ element is $\mathrm{y}_{\mathbf{n j}}^{\prime}$. Equation (4) implicitly treats the inferred choices as actual observed choices and overlooks errors in inference during estimation. Most inference algorithms will predict for a given observation the probability of occurrence associated with every possible outcome. When constructing the variable $\mathrm{y}_{\mathrm{nj}}^{\prime}$, the outcome that has the greatest probability is assumed to have taken place and the probability distribution across outcomes is subsequently discarded. While such an approach is standard practice, it risks introducing measurement error into the choice model, which may result in biased parameter estimates. To account for this error during parameter estimation, we propose maximizing the following weighted likelihood function that makes use of the probability distribution across all possible outcomes: 


$$
\begin{aligned}
\mathrm{L}\left(\boldsymbol{\beta} \mid \mathbf{y}^{\prime}, \mathbf{X}\right) & =\prod_{n} \sum_{j} \mathrm{P}\left(\mathrm{y}_{\mathrm{nj}}^{\prime}=1\right) \mathrm{P}\left(\mathbf{y}_{\mathbf{n}} \mid \boldsymbol{\beta} ; \mathbf{X}_{\mathbf{n}}, \mathrm{y}_{\mathbf{n j}}^{\prime}=1\right) \\
& =\prod_{n} \sum_{\mathrm{j}} \mathrm{P}\left(\mathrm{y}_{\mathrm{nj}}^{\prime}=1\right) \mathrm{P}\left(\mathrm{y}_{\mathrm{nj}}=1 \mid \boldsymbol{\beta} ; \mathbf{X}_{\mathbf{n}}\right) \\
& =\prod_{\mathrm{n}} \sum_{\mathrm{j}} \mathrm{P}\left(\mathrm{y}_{\mathbf{n j}}^{\prime}=1\right)\left[\frac{\exp \left(\boldsymbol{\beta}^{\prime} \mathbf{X}_{\mathbf{n j}}\right)}{\sum_{\mathbf{j}^{\prime}} \exp \left(\boldsymbol{\beta}^{\prime} \mathbf{X}_{\mathbf{n} \mathbf{j}^{\prime}}\right)}\right]
\end{aligned}
$$

, where $\mathrm{P}\left(\mathrm{y}_{\mathrm{nj}}^{\prime}=1\right)$ is the class-specific probability that the inference algorithm predicts that individual $\mathrm{n}$ chose travel mode j. For our experiment, if $y_{n i}=1$, then $P\left(y_{n j}^{\prime}=1\right)$ corresponds to the $(i, j)^{\text {th }}$ element of the confusion matrix. Note that as the inference accuracy approaches $100 \%$, equation (5) converges to equation (4). Ideally, the analyst should jointly estimate the inference and choice model, but for practical reasons that isn't always possible. It is usually not straightforward to recast classifiers employed for inference as probabilistic models that can subsequently be estimated using maximum likelihood estimation. Most classifiers continue to be estimated using metrics derived from information theory that differ from the probabilistic framework employed by models of travel and activity behavior. But more importantly, the inference algorithm is often trained on a dataset where the explanatory variables used in the choice model are not available and/or collected. The alternative, as represented by equation (5), is to estimate the inference model independently, and to treat the outcomes predicted by the inference model as stochastic variables in the choice model, marginalizing over the estimated distribution. Such an approach leads to consistent but inefficient estimates in cases where it can be assumed that the features used to train the inference model are either independent of the explanatory variables used in the choice model or they don't affect the choice outcome. If the two sets of variables are correlated and the features do influence observed choice, as will likely be the case in reality, then estimates from the choice model may still suffer from omitted variable bias.

All models are estimated in Python using an implementation of the BFGS algorithm contained in the SciPy library (Jones et al. 2014). For each of the three Monte Carlo experiments, described in greater 
detail in the subsections that follow, we compare parameter estimates from maximizing the likelihood functions given by both equation (4) and equation (5) with the true underlying values.

\subsection{Monte Carlo Experiment 1}

For our first experiment, we choose a very parsimonious specification for our RUM model of travel mode choice behavior. The utility of each of the four travel modes is specified as a linear function of the total travel time and cost incurred by that travel mode:

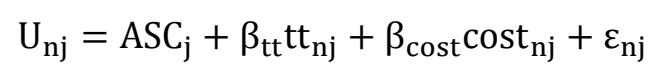

, where $\mathrm{tt}_{\mathrm{nj}}$ and $\operatorname{cost}_{\mathrm{nj}}$ denote the total travel time and cost incurred by travel mode $\mathrm{j}$ on decision-maker $\mathrm{n}$, respectively; the model parameters $\mathrm{ASC}_{\mathrm{j}}, \beta_{\mathrm{tt}}$ and $\beta_{\text {cost }}$ denote the alternative-specific constant and the mean sensitivities to travel time and cost, respectively; and $\varepsilon_{\mathrm{nj}}$ denotes the stochastic component of the utility of travel mode $\mathrm{j}$ for decision-maker $\mathrm{n}$. Table 1 enumerates the mode-specific distributions used for each of the variables and Table 2 enumerates the selected parameter values (utility is assumed to be measured in utils, a fictitious microeconomic construct). In terms of the variables, the distributions are chosen to be realistic of travel times and costs that would be incurred by each of the four travel modes for intra-city commute travel in a mid-sized metropolitan region in the United States (McKenzie \& Rapino 2011). In terms of the parameters, values are chosen to suffice the three conditions mentioned in the introduction to Section 2. For example, the value of travel time, as denoted by the ratio of the parameters $\beta_{\mathrm{tt}}$ and $\beta_{\text {cost }}$, is set to be $20 \$ / \mathrm{hr}$, which for an individual working 40 hours a week corresponds to an annual salary of approximately $\$ 42,000$. For comparison, the minimum wage rate in California is $9 \$ / \mathrm{hr}$ and the median per capita income for California as per the 2011 American Community Survey is $\$ 29,634$.

The stochastic component $\varepsilon_{\mathrm{nj}}$ for all travel modes and decision-makers is generated using a Gumbel distribution with location zero and scale one.

\section{$<$ Insert Table 1 roughly around here $>$}


$<$ Insert Table 2 roughly around here $>$

<Insert Figure 2 roughly around here $>$

Errors in travel mode inference are simulated using symmetric confusion matrices, such as the one shown in Figure 2, that make two simplifying assumptions: (1) mode-specific accuracies are assumed to be the same for all travel modes, and therefore equal to the average accuracy of the algorithm, denoted as $\alpha$ in Figure 2; and (2) for any chosen travel mode, the probability of inferring one of the non-chosen travel modes is assumed to be the same regardless of the chosen and non-chosen travel mode, and is denoted as $\gamma$ in Figure 2 (where $\alpha+3 \gamma=1$ ). Note that this is rarely the case in practice. Most studies in the literature report higher average accuracies for trips made by non-motorized modes, i.e. bike and walk, and difficulties differentiating between motorized modes, i.e. car and transit (Wolf et al. 2014). While our assumptions regarding both the utility specification and the accuracy of the inference algorithm are admittedly simple, they make for a good starting point for any kind of analysis. In subsequent experiments, we will relax these assumptions and see how they affect the estimation results. 100 datasets each are generated for 100, 1000 and 10000 pseudo-observed decision-makers hypothesized to behave according to the decision-making process described above. For each of these datasets, errors in inference are simulated assuming average accuracies of the inference algorithm, or $\alpha$, between $60 \%$ and $100 \%$, implemented in 5\% increments. In all, a total of 2700 datasets are simulated. For each of these datasets, we estimate the model parameters by maximizing the unweighted likelihood function given by equation (4) and the weighted likelihood function given by equation (5). The impact of inference errors is assessed by comparing these estimates with the true values.

\section{<Insert Figure 3 roughly around here $>$}

We begin by comparing estimates for the value of travel time, as denoted by the ratio of the parameters $\beta_{\mathrm{tt}}$ and $\beta_{\mathrm{cost}}$, from the unweighted likelihood function. Note that the true value of time is $20 \$ / \mathrm{hr}$. Figure 3 shows a boxplot where, for each of the 100 datasets belonging to a particular combination of the number 
of observations and the average accuracy of the inference algorithm, the horizontal line at the center of the box denotes the median estimate, the horizontal edges of the box denote the 25 th and 75 th percentile estimates, and the ends of the vertical line denote the minimum and maximum estimates.

For the model specification at hand, the bias in estimates expectedly decreases as the average accuracy of the inference algorithm increases, eventually converging to the true values at an accuracy of $100 \%$. For 100 observations, this trend is not always consistent, particularly at lower average accuracies. For example, the median value of time at $60 \%$ is closer to the true value than the median value of time at $70 \%$. However, this is likely due to the small number of observations introducing greater variability in estimation results. In general, the variability in estimates decreases as the number of observations increase, but the magnitude of bias appears to be independent of the number of observations, and surprisingly large. The median estimate for value of time for 10000 observations and an inference accuracy of $80 \%$ is $12.9 \$ / \mathrm{hr}$, off by $35 \%$ from the true value of $20 \$ / \mathrm{hr}$. Even at higher accuracies, such as $95 \%$, the median estimate for 10000 observations is $17.3 \$ / \mathrm{hr}$, off by $14 \%$. The magnitude of bias will vary from dataset to dataset. The purpose of the Monte Carlo experiment is not to determine an absolute range, but to demonstrate the variability in estimates that can be expected from inaccuracies in inference.

\section{$<$ Insert Figure 4 roughly around here>}

Next, we compare estimates for the value of travel time from the weighted likelihood function. Similar to Figure 3, Figure 4 shows a boxplot of the estimates for value of time recovered from each of the 100 datasets belonging to a particular combination of the number of observations and the average accuracy of the inference algorithm. The vertical lines and boxes running through the plots indicate large standard errors in the parameter estimates, indicating a failure in the optimization routine to consistently recover unbiased estimates. However, the frequency with which these vertical lines and boxes appear decreases as both the number of observations and the accuracy of inference increase, and for large datasets with high inference accuracies the optimization routine is able to recover unbiased estimates for the value of time 
consistently. For example, with 100 observations anything less than complete accuracy is unable to recover parameter estimates consistently, but with 10000 observations the parameter estimates can be recovered consistently with average accuracies of $90 \%$ and above.

A comparison between estimates from the unweighted and weighted likelihood function confirms that maximum unweighted likelihood estimation yields efficient but inconsistent estimates, whereas maximum weighted likelihood estimation provides consistent but inefficient estimates. The choice between the two estimation methods comes down to a tradeoff between bias and variance. For a given dataset, the appropriate method will depend upon the sample size, the accuracy of the inference algorithm and the desired complexity of the travel demand model specification. For example, for the model specification at hand and an inference algorithm that produces symmetric confusion matrices of the form given by Figure 2, for a sample size of 10000 observations, our results suggest that maximum likelihood estimation ought to be preferred when the average accuracy of the inference algorithm is below $90 \%$, and maximum weighted likelihood estimation ought to be preferred when the average accuracy is above $90 \%$.

These results indicate that increases in the amount of data that can potentially be retrieved using newer technologies are often offset by the loss in quality incurred by inaccuracies in inference. For example, Figure 4 suggests that the same information that could reliably be retrieved from 100 high-quality observations could potentially need 10000 observations and more, depending upon the accuracy of inference and the tolerance for bias. Conversely, one could argue that with sufficiently large datasets, e.g. location data retrieved from sparse sources such as cellphone towers that cannot provide the same accuracy in inference as denser sources such as GPS sensors, the parameters of interest could still be recovered with a high degree of precision. As before, the purpose of this analysis is not to develop normative guidelines on when to use low quality big data and when to forego it in favor of high quality 
'small' data ${ }^{2}$, but to illustrate the kinds of issues that might be encountered when working with these larger datasets, and the ways in which they can be controlled for, if only partially.

\subsection{Monte Carlo Experiment 2}

For our second experiment, we choose a more realistic specification for our RUM model of travel mode choice behavior. The utility of each of the four travel modes is specified as a linear function of the invehicle travel time, walking time, biking time, waiting time and cost incurred by that travel mode:

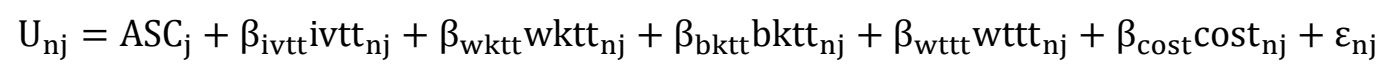

, where ivtt ${ }_{n j}, w_{k t t}$, bktt ${ }_{n j}, w_{t t t}$ and cost $_{n j}$ denote the in-vehicle travel time, walking time, biking time, waiting time and cost incurred by travel mode $\mathrm{j}$ on decision-maker $\mathrm{n}$, respectively; the model parameters $\mathrm{ASC}_{\mathrm{j}}, \beta_{\mathrm{ivtt}}, \beta_{\mathrm{wktt}}, \beta_{\mathrm{bktt}}, \beta_{\mathrm{wttt}}$ and $\beta_{\text {cost }}$ denote the alternative-specific constant and the mean sensitivities to in-vehicle travel time, walking time, biking time, waiting time and cost, respectively; and $\varepsilon_{\mathrm{nj}}$ denotes the stochastic component of the utility of travel mode $\mathrm{j}$ for decision-maker $\mathrm{n}$.

Typically, a travel mode choice model would include a number of additional variables related to the socioeconomic characteristics of the individual, the built environment of the trip origin and destination, etc. While there is no limit to the variables that can be included, we wish to strike a balance between realism and parsimony. We do not want our model specification to be entirely unrepresentative of the state of practice. At the same time, we do not want to have so many model parameters that it becomes unfeasible to examine the impact of inference errors on any one parameter. The results from our experiments are meant to be illustrative. If the reader wishes to assess the impact of inference errors on a

\footnotetext{
${ }^{2}$ Note that data collected through traditional travel diary surveys has errors of its own, and may be no more 'high quality' than data collected from GPS-based surveys. By high quality data, big or small, we denote an ideal: data that is free from any kind of measurement error.
} 
more complex model specification, the Monte Carlo framework described here can easily be extended to include such a specification.

\section{$<$ Insert Table 3 roughly around here $>$}

\section{$<$ Insert Table 4 roughly around here $>$}

Table 3 enumerates the mode-specific distributions used for each of the variables and Table 4 enumerates the selected parameter values. As in the case of the first experiment, the distributions for each of the explanatory variables are chosen to reflect the times and costs incurred by each of the four travel modes for intra-city commute travel in a mid-sized metropolitan region in the United States (McKenzie \& Rapino 2011) and the parameter values are chosen to reflect marginal rates of substitution reported in the literature. For example, time spent waiting is defined to be more onerous than time spent traveling (Ory \& Mokhtarian 2009), in our case by a factor of 2. Readers can check for themselves that ratios between other parameters make intuitive sense as well. As was the case in Section 2.1, the stochastic component $\varepsilon_{\mathrm{nj}}$ for all travel modes and decision-makers is generated using a Gumbel distribution with location zero and scale one.

Errors in travel mode inference are simulated using the same symmetric confusion matrices used in the first experiment. In our third and final experiment, we shall relax this assumption as well. 100 datasets each are generated for 100, 1000 and 10000 pseudo-observed decision-makers and average accuracies of the inference algorithm between $60 \%$ and $100 \%$, implemented in 5\% increments, resulting in a total of 2700 datasets. For each of these datasets, model parameters are estimated using both the unweighted and weighted likelihood functions and subsequently compared to the true values.

\section{$<$ Insert Figure 5 roughly around here $>$}

We begin by comparing estimates for the value of in-vehicle travel time, value of walking time, value of biking time and value of waiting time, as denoted by the ratio of the respective parameters with $\beta_{\text {cost }}$, 
from the unweighted likelihood function. Note that the true value of in-vehicle travel time, walking time, biking time and waiting time, implicitly assumed under the parameter values enumerated in Table 4, are $20 \$ / \mathrm{hr}, 10 \$ / \mathrm{hr}, 30 \$ / \mathrm{hr}$ and $40 \$ / \mathrm{hr}$, respectively. Figure 5 shows a boxplot depicting the median, 25th and 75th percentile, and minimum and maximum estimates for each of these marginal rates of substitution for each of the 100 datasets belonging to a particular combination of the number of observations and the average accuracy of the inference algorithm.

There are a number of similarities between Figure 5 and Figure 3, the analogous boxplot for the first experiment. The bias in estimates decreases as the average accuracy of the inference algorithm increases, the variability in estimates decreases as the number of observations increases, and the magnitude of bias appears to be independent of the number of observations. However, there are two key differences as well. First, the relative magnitude of bias appears to be larger. For example, the median estimates for value of in-vehicle, walking and biking time for 10000 observations and an inference accuracy of $80 \%$ are $9.7 \$ / \mathrm{hr}$, $3.2 \$ / \mathrm{hr}$ and $6.9 \$ / \mathrm{hr}$, respectively, off from their true values by $52 \%, 68 \%$ and $77 \%$, respectively. The median estimates for value of waiting time though seem to be closer to the true values. For example, the median estimate for 10000 observations and an inference accuracy of $80 \%$ is $48.0 \$ / \mathrm{hr}$, off from the true value by $20 \%$. And second, there is greater variability in the estimates. For a sample size of 100 observations, regardless of the average accuracy of the inference algorithm, the maximum estimate for any of the marginal rates of substitution seems to be unreasonably large. As the number of observations increase, the variability does indeed decrease. However, even with 10000 observations, for average accuracies of the inference algorithm below $75 \%$, the maximum estimate for any of the marginal rates of substitution continues to be unreasonably large. These differences in results between the two experiments are not wholly unsurprising, given that the model specification for the second experiment is more complex than the model specification for the first experiment.

\section{<Insert Figure 6 roughly around here>}


Next, we compare estimates for the same marginal rates of substitution from the weighted likelihood function using analogous boxplots, shown in Figure 6. Similar to the other boxplots, the vertical lines and boxes running through the plots indicate a high degree of variability in the parameter estimates. To our great surprise though, these vertical lines and boxes appear in Figure 6 much less frequently than they do in Figure 4, the analogous boxplot for the first experiment, despite (or perhaps because of?) the second experiment using a more complex travel mode choice model specification than the first. When we first saw these plots and how they compare with those in Figure 4, we assumed the difference must be a peculiarity arising out of the variable distributions and model parameter values used for the two experiments. However, no matter how we changed the variable distributions or the model parameter values for either experiment, the results stayed the same: when maximizing the weighted likelihood function to recover parameter values, estimates from the model with alternative-specific parameters were always less biased than estimates from the model with generic parameters, even though the latter has fewer total parameters. In general, it appears that alternative-specific parameters are easier to estimate consistently than generic parameters, and consequently more complex model specifications are not necessarily harder to estimate consistently than less complex model specifications. These results further substantiate the argument first made in Section 2.1 that with a sufficiently large number of observations, even if the inference algorithm is not very accurate, the parameters of interest could still potentially be recovered with a high degree of precision.

\subsection{Monte Carlo Experiment 3}

For our third and final experiment, we use the same specification for our RUM model of travel mode choice behavior as in the second experiment, given by equation (7) and Tables 3 and 4. However, unlike the second experiment, we simulate errors in travel mode inference using confusion matrices that are more realistic. Figure 7 shows the general form, loosely based on the confusion matrix cited by NCHRP report 775 for the best practices travel mode inference algorithm shown in Figure 1. The following four conditions must be satisfied by the matrix: 


$$
\begin{aligned}
& 0.4 \gamma+0.6 \delta=\alpha \\
& \gamma=\min \left(\frac{8}{5} \delta, 1\right) \\
& \gamma+4 \rho=1 \\
& \delta+10 \sigma=1
\end{aligned}
$$

, where $\alpha$ denotes the desired average accuracy of the inference algorithm. Equation (8) can be derived from the fact that the average mode shares resulting from the RUM model specification are as follows: $60 \%$ choose car and $40 \%$ choose one of the other three travel modes. Equation (9) ensures that the ratio of the mode-specific accuracies for car and transit is the same as that in the confusion matrix cited in NCHRP report 775. Equations (10) and (11) can be derived from simple probability axioms. Table 5 shows, for a given average accuracy of the inference algorithm, the corresponding values for the other elements of the confusion matrix resulting from equations (8)-(11). These values are motivated by two trends consistently noted in the literature on travel mode inference (see, for example, Wolf et al. 2014): (1) non-motorized modes, such as walk and bike, are easier to identify; and (2) motorized modes, such as car and transit, are harder to distinguish.

$<$ Insert Figure 7 roughly around here $>$

<Insert Table 5 roughly around here $>$

As before, 100 datasets each are generated for 100, 1000 and 10000 pseudo-observed decision-makers and average accuracies of the inference algorithm between $60 \%$ and $100 \%$, implemented in $5 \%$ increments, resulting in a total of 2700 datasets. For each of the datasets, model parameters are estimated using the unweighted and weighted likelihood functions and subsequently compared to the true values.

$<$ Insert Figure 8 roughly around here> 
Figure 8 shows a boxplot depicting the median, 25th and 75th percentile, and minimum and maximum estimates for the marginal rates of substitution between each of the travel time components and travel cost for each of the 100 datasets belonging to a particular combination of the sample size and inference accuracy as estimated using the unweighted likelihood function. There are two key observations to be made. First, in comparison to Figure 5, the corresponding boxplot for the second experiment, the magnitude of bias appears to be smaller. While this may seem surprising at first, note from Table 5 that even at low average accuracies of the inference algorithm, the mode-specific accuracies for walk, bike and transit, as given by $\gamma$, are quite high, which helps in the estimation of parameters specific to these alternatives. Second, the slope of the curve for each of the four marginal rates of substitution seems to bend downwards around the $80 \%$ mark. This is perhaps most clearly discernible in the plots corresponding to a sample size of 10000 observations. Again, this can be explained using the values enumerated in Table 5. When the average accuracy of the inference algorithm is $80 \%$ or more, the modespecific accuracies for walk, bike and transit are $100 \%$. In other words, all trips made by walk, bike or transit are correctly identified, and the bias in parameter estimates comes solely from trips made by car incorrectly classified as having been made by one of the other three travel modes. When the average accuracy of the inference algorithm decreases beyond $80 \%$, the performance of the inference algorithm with regards to trips made by all travel modes starts to decrease. This introduces measurement error in the choice model at a higher rate than before. As a consequence, the bias in parameter estimates, as judged by the slope of the curve, increases at a proportionally higher rate as well.

\section{<Insert Figure 9 roughly around here>}

Figure 9 shows the analogous boxplot for the weighted likelihood function. In comparison to Figure 6, the corresponding boxplot for the second experiment, there is one notable difference: at accuracies less than $100 \%$, regardless of the number of observations, the parameter estimates appear to be biased. These findings corroborate the assertion, first made in the introduction to Section 2, that in cases where the error in inference is not purely stochastic but is correlated with observed choices, as is the case here, estimates 
from the weighted likelihood function may still be biased. That being said, the magnitude of bias is much smaller than in the case of estimates from the unweighted likelihood function. For example, for 10000 observations and an average accuracy of $90 \%$, the median estimate for value of in-vehicle travel time as recovered using the unweighted and weighted likelihood functions are $18.1 \$ / \mathrm{hr}$ and $19.4 \$ / \mathrm{hr}$, off by $9 \%$ and $3 \%$ from the true value of $20 \$ / \mathrm{hr}$, respectively. Similarly, the variability in estimates for the weighted likelihood function, as denoted by the length of the vertical lines, is smaller than the variability in estimates for the unweighted likelihood function. In general, the weighted likelihood function ought still to be preferred at higher average accuracies and larger sample sizes. However, with asymmetric confusion matrices that risk correlation between the measurement error and the choice outcome, the benefits of estimating parameters using the weighted likelihood function will be smaller than in the case of symmetric confusion matrices where the measurement errors are purely stochastic and independent of the choice outcome.

\section{Case Study: GPS-based Survey in the San Francisco Bay Area, United States}

In this section, we corroborate findings from Section 2 using real data collected from smartphone users living in the San Francisco Bay Area, United States through the means of an app called E-Mission. The app is being developed by a team of researchers at the University of California (UC), Berkeley. One of the objectives of E-Mission is to collect all the information that is usually collected by travel diary surveys, but with minimal input from the smartphone user. For more details about the app, the reader is referred to Shankari et al. (2014). E-Mission records two pieces of information. First, the app records the location and motion of the smartphone user at regular time intervals, using GPS, Wi-Fi and accelerometer sensors on the phone. This data is fed as input to a first set of inference algorithms that have been trained to separate trips from activities. For an inferred trip, the time-stamped location traces and accelerometer readings that constitute the trip are used to calculate a set of features that are subsequently fed as input to a second set of inference algorithms that have been trained to infer the travel mode(s) used to complete 
the trip (based on the travel mode inference algorithms developed by Zheng et al. 2010). Second, the app disseminates a prompted-recall survey to the smartphone user through the mechanism of a notification, pushed to the smartphone within two hours of when the trip was completed, as inferred by the algorithm, The survey asks the user to indicate whether the trip detected by the inference algorithm was indeed made, and if it was, what were the travel modes used. As is standard practice, this information is treated as ground truth and is used to train and/or validate the inference algorithms ${ }^{3}$.

In all, data from 3381 trips collected from 45 smartphone users over a three-month period in 2014 is used for our analysis. Though demographic information was not collected from study participants as part of the experiment, anecdotal evidence indicates that most participants are graduate students at UC, Berkeley, with a handful of undergraduate students, faculty and researchers, also employed at the university, making up the remainder of the sample. The mode shares for trips across the sample are as follows: $31 \%$ walk, $41 \%$ bike, $18 \%$ drive and $11 \%$ take transit. While neither the sampled population nor their travel mode choice patterns are representative of the general population living in the Bay Area (and the Bay Area itself is not representative of the United States at large), the experiment is meant to be illustrative of possible problems that might be encountered when working with GPS-based surveys for the estimation of travel demand models. The specific nature of the problems will vary, depending upon the characteristics of the sampled population and their travel patterns, but it is hoped that the experiment can offer some general insights that might be useful regardless of context.

\footnotetext{
${ }^{3}$ Without getting into deeper ontological debates, ground truth denotes what actually, objectively and inarguably happened, not what may have been reported by the study participant through personal recall surveys, nor what may have been inferred by algorithms using GPS data. In the absence of more reliable alternatives, we are forced to accept self-reported information as ground truth, but that is not to insinuate that self-reported information is always a good measure of ground truth. Differences between ground truth and self-reported information may be sources of additional error, but unfortunately there was no way to control for it within the design of the experiment.
} 
Our experiment design is as follows: First, for each trip in the dataset, we use the location traces and accelerometer readings to construct a vector of features comprising trip attributes such as speed, acceleration, heading change, etc. Second, we employ different subsets of the full set of features and the ground truth collected by E-Mission to train classification and regression tree (CART) learning algorithms for travel mode inference with average accuracies between $60 \%$ and $100 \%$. CART algorithms are part of the broader family of decision tree learning algorithms that "construct binary trees using the feature and threshold value that yield the largest information gain at each node". All CART algorithms used in this study were calibrated in Python using the Scikit-learn library (Pedregosa et al. 2011). Though the mode-specific accuracies varied based on the average accuracy of the algorithm, in general, consistent with trends noted in the literature, our algorithm was found to perform better for non-motorized travel modes and had a harder time distinguishing between motorized travel modes. Third, for each trip in the sample, we generate the predicted outcome and the predicted probability distribution across all outcomes, as predicted by each of the CART algorithms trained in the previous step (we do not split the data into calibration and validation sets, using the classifier trained on trips where we know the outcomes to predict outcomes for those same trips as if we didn't know what the outcomes were ${ }^{4}$ ). Fourth, we derive the travel times and costs incurred by different travel modes for all trips in the sample using transportation

\footnotetext{
${ }^{4}$ Though this results in algorithms that have been overfitted to the calibration dataset, it isn't a concern because our objective here is not to make predictions for a different dataset where we don't have the ground truth, but to simulate errors in inference for the calibration dataset where we do have the ground truth, or an acceptably close approximation to it (and therefore have a measure of the inference error with respect to that specific dataset). We could simulate errors using just the confusion matrix, as we did in the case of the Monte Carlo experiments. However, we want the setup of our experiment design to be as realistic as possible, and consequently, we wish to simulate the mechanics of how an inference algorithm would work in practice. Overfitting to the calibration dataset is necessary because we wish to evaluate the impact of errors in inference across all possible values. If we didn't overfit, we wouldn't be able to evaluate the impact beyond an average accuracy of $86 \%$, the maximum average accuracy that we were able to achieve for the dataset, as measured using a validation sample.
} 
network skims from the San Francisco Metropolitan Transportation Commission (SF MTC). And finally, we use the predictions from the inference algorithms and the level-of-service attributes derived from the skims to estimate multinomial logit models of travel mode choice, using both maximum unweighted likelihood estimation and maximum weighted likelihood estimation. For each trip, the decision-maker is hypothesized to have at most four travel modes to choose from: walk, bike, car and public transit, and the systematic component of the utility of each travel mode is defined as a linear function of the travel time and cost incurred by that travel mode.

\section{<Insert Figure 10 roughly around here $>$}

Figure 10 plots estimates for the value of time, as recovered by the multinomial logit model from both maximum unweighted likelihood estimation and maximum weighted likelihood estimation, as a function of the average accuracy of the decision tree used for travel mode inference. The value of time estimated when the inference algorithm has $100 \%$ accuracy is $27.6 \$ / \mathrm{hr}$. For our analysis, we will treat this as the true value, using it as a baseline for measuring bias. Figure 10 reveals a number of key trends. At lower average accuracies, the value of time recovered from maximum unweighted likelihood estimation is closer to the true value than that recovered from maximum weighted likelihood estimation, consistent with the high standard errors observed at lower average accuracies for the latter in the Monte Carlo experiment. At higher average accuracies, maximum weighted likelihood estimation performs better than maximum unweighted likelihood estimation, consistent once again with findings from the Monte Carlo experiment. However, both estimation procedures fall apart when the average accuracy is $85 \%$, with the value of time tending towards positive infinity for maximum unweighted likelihood estimation and negative infinity for maximum weighted likelihood estimation. This is likely due to incorrect inferences for a handful of trips that appear to exert a disproportionate influence on the estimation results. We could have removed these trips from our analysis, as would have to be the course to take in reality, but we decided to include them in our analysis to illustrate potential problems that the analyst may face when working with low-quality data. As mentioned in Section 2.1, the choice between the two estimation 
methods comes down to a tradeoff between bias and variance: maximum unweighted likelihood estimation yields efficient but inconsistent estimates, whereas maximum weighted likelihood estimation provides consistent but inefficient estimates. In our case, for a sample size of 3381 trips and a relatively simple travel mode choice model specification with generic parameters, our results suggest that maximum unweighted likelihood estimation ought to be preferred when the average accuracy of the inference algorithm is below $85 \%$, and maximum weighted likelihood estimation ought to be preferred when the average accuracy is above $85 \%$.

Regardless of the chosen estimation method, even at high average accuracies the bias in estimates is sizeable. For example, at an average accuracy of $95 \%$, the values of time recovered by maximum unweighted likelihood estimation and maximum weighted likelihood estimation are $34.5 \$ / \mathrm{hr}$ and $31.3 \$ / \mathrm{hr}$, respectively, off by $25 \%$ and $13 \%$ from the true value, respectively. These results serve as a cautionary warning against the use of travel diary data inferred from GPS-based surveys for travel demand analysis. Unless the average accuracy of the inference algorithm is close to $100 \%$, the magnitude of bias in parameter estimates may render the use of such data for model development infeasible.

In practice, no inference algorithm will ever be $100 \%$ accurate, and data collected passively through mobile sensors or social media platforms may always need to be augmented with ground truth for it to be usable. However, as the average accuracy of the inference algorithm deteriorates, the number of observations for which ground truth is needed will increase, as will the consequent burden on study participants. To get an estimate of the trade-off between estimation accuracy and participant burden, we perform the following experiment with each of the inference algorithms trained previously. For trips where the outcome predicted by the inference algorithm has a probability of occurrence above $90 \%$, we use the predicted probability distribution in calculating the weighted likelihood function. For trips where the outcome predicted by the inference algorithm has a probability of occurrence under $90 \%$, we use the ground truth in calculating the weighted likelihood function, assuming that in these cases the study 
participant can be asked what they did, as they would be in a traditional travel diary survey and assuming that this information is accurate.

\section{<Insert Figure 11 roughly around here $>$}

Figure 11 plots both the value of time recovered from maximizing the weighted likelihood function thus constructed, and the percentage of observations for which study participants would potentially be required to provide ground truth under the scheme described above, as a function of the average accuracy of the inference algorithm. As is apparent from the plot, even at low accuracies, estimates are within $2-3 \%$ of the true value (though again, at an average accuracy of $85 \%$, the estimation routine breaks down). However, as the average accuracy decreases, the burden on study participants increases. For example, at low average accuracies between $60 \%$ and $70 \%$, using $90 \%$ as the threshold to determine when to ask for ground truth and when to rely on the inference algorithm, we would need to ask for ground truth for more than $80 \%$ of the trips. However, at higher accuracies, the burden is more acceptable. For example, when the average accuracy of the inference algorithm is $94 \%$, and the threshold is still $90 \%$, ground truth is needed for only $15 \%$ of the trips, but the bias in our estimate for value of time is less than $0.1 \%$. In general, the analyst can decide upon an appropriate threshold for the partial collection of ground truth based on the levels of participant burden and errors in estimation that are acceptable given the objectives of the study.

\section{Conclusions}

The last few years have been witness to great excitement over big data and its potential to address a multitude of societal problems, within transportation engineering and without, on an unprecedented scale and level of detail. The National Science Foundation's call for research proposals in 2014 on "Critical Techniques and Technologies for Advancing Big Data Science and Engineering”, the Transportation Research Board's call for papers on "Big Data, ICTs, and Travel Demand Models" for its 93rd Annual Meeting and Transportation Research Part C's recent call for papers on "Big Data in Transportation and 
Traffic Engineering" reflect some of the ongoing interest. With regards to travel demand analysis, attention has centered on the development of fully automated GPS-based surveys that can allow for the collection of travel diary data from a greater subset of the population over a longer period of time at a fraction of the cost incurred by more traditional survey methods. The collection of richer travel diary datasets can lead to significant advances in our understanding of travel behavior and consequently, our ability to design transportation systems that serve the immediate needs of the population and satisfy longterm societal objectives.

GPS-based surveys record an individual's location over time, augmented in some cases by information from additional sensors, such as accelerometers and Wi-Fi devices. However, certain vital inputs to the travel demand modeling process, such as the travel mode(s) taken by the individual to make a trip or the purpose of the trip, must necessarily be inferred from this data. Errors in inference can compromise the quality of the data thus collected, raising questions about the validity of travel demand models estimated using this data. In an attempt to address these questions, this study examined the impact that errors in inference can have on estimation results and proposed ways in which they can be controlled for during data collection and model estimation. We used simulated datasets to compare performance across different sample sizes, inference accuracies and estimation methods. Findings were corroborated using real data collected from smartphone users living in the San Francisco Bay Area, United States. Results indicate that the benefits of using GPS-based surveys will vary significantly, depending upon the sample size of the data, the accuracy of the inference algorithm and the desired complexity of the travel demand model specification. If the data is truly big enough, the quality of inference may not matter. But in many cases, gains in volume could potentially be neutralized by losses in quality. For example, a Monte Carlo experiment finds that a relatively parsimonious model of travel mode choice behavior that could reliably be estimated using 100 high-quality observations could need 10000 observations and more, depending upon the accuracy of the inference algorithm. In practice, no algorithm will ever guarantee complete accuracy. For data from GPS-based surveys to still be useful for travel demand analysis, it will need 
either to be incredibly big, or it will need to be supplemented with data that can be treated as a reliable source of ground truth.

\section{Acknowledgements}

This research was funded in part by the Jim Gray Fellowship and in part by the NSF ActionWebs CPS0931843. Jim Gray, who did pioneering work on the management of large amounts of data, disappeared while sailing in the San Francisco Bay in 2007. We hope that he would have found this exploration into data sizes and accuracies interesting. We wish to thank Mogeng Yin, Shanthi Shanmugam and Ryan Lei for their help in developing E-Mission, the smartphone app used by this study for data collection. And finally, we wish to thank all the people, anonymous or known to the authors, who reviewed this study. Their helpful comments and constructive criticisms contributed much in terms of improving the presentation of our ideas. 


\section{List of References}

Bachman, W, Oliveira, M, Xu, J, \& Sabina, E 2012, 'Using household-level GPS travel data to measure regional traffic congestion,' 91st Annual Meeting of the Transportation Research Board, Washington, D. C.

Bohte, W \& Maat, K 2009, 'Deriving and validating trip purposes and travel modes for multi-day GPS-based travel surveys: A large-scale application in the Netherlands,' Transportation Research Part C: Emerging Technologies, vol. 17 , no. 3, pp. 285-297.

Bolbol, A, Cheng, T, Tsapakis, I, \& Haworth, J 2012, 'Inferring hybrid transportation modes from sparse GPS data using a moving window SVM classification,' Computers, Environment and Urban Systems, vol. 36, no. 6, pp. $526-537$.

Bolbol, A, Cheng, T, \& Tsapakis, I 2014, 'A spatio-temporal approach for identifying the sample size for transport mode detection from GPS-based travel surveys: A case study of London's road network,' Transportation Research Part C: Emerging Technologies, vol. 43, pp. 176-187.

Bricka, S \& Bhat, C 2007, 'Comparative analysis of Global Positioning System-based and travel survey-based data,' Transportation Research Record: Journal of the Transportation Research Board, vol. 1972, pp. 9-20.

Carrel, A, Lau, PSC, Mishalani, RG, Sengupta, R, \& Walker, JL 2015, 'Quantifying transit travel experiences from the users' perspective with high-resolution smartphone and vehicle location data: Methodologies, validation, and example analyses,' Transportation Research Part C: Emerging Technologies, in press.

Feng, T \& Timmermans, HJ 2013, 'Transportation mode recognition using GPS and accelerometer data,' Transportation Research Part C: Emerging Technologies, vol. 37, pp. 118-130.

Gonzalez, P, Weinstein, JS, Barbeau, SJ, Labrador, MA, Winters, PL, Georggi, NL, \& Perez, R 2008, 'Automating mode detection using neural networks and assisted GPS data collected using GPS-enabled mobile phones," 15th World Congress on Intelligent Transportation Systems.

Hasan, S \& Ukkusuri, SV 2014, 'Urban activity pattern classification using topic models from online geo-location data,' Transportation Research Part C: Emerging Technologies, vol. 44, pp. 363-381.

Hemminki, S, Nurmi, P, \& Tarkoma, S 2013, 'Accelerometer-based transportation mode detection on smartphones,' in Proceedings of the 11th ACM Conference on Embedded Networked Sensor Systems, Rome, Italy, p. 13. 
Jones, E, Oliphant, T, \& Peterson, P 2014, ‘SciPy: Open source scientific tools for Python,' viewed 24 April 2015, http://www.scipy.org/.

McFadden, D 2001, ‘Economic choices,' The American Economic Review, vol. 91, no. 3, pp. 351-378.

McKenzie, B \& Rapino, M 2011, 'Commuting in the United States: 2009,' US Department of Commerce, Economics and Statistics Administration, US Census Bureau.

Oliveira, M, Vovsha, P, Wolf, J, Birotker, Y, Givon, D, \& Paasche, J 2011, 'GPS-assisted prompted recall household travel survey to support development of advanced travel model in Jerusalem,' 90th Annual Meeting of the Transportation Research Board, Washington, DC.

Ory, DT \& Mokhtarian, PL 2009, 'Modeling the structural relationships among short-distance travel amounts, perceptions, affections, and desires,' Transportation Research Part A: Policy and Practice, vol. 43, no. 1, pp. 2643.

Pedregosa, F, Varoquaux, G, Gramfort, A, Michel, V, Thirion, B, Grisel, O, \& Duchesnay, É 2011, 'Scikit-learn: Machine learning in Python,' The Journal of Machine Learning Research, vol. 12, pp. 2825-2830.

Pereira, F, Carrion, C, Zhao, F, Cottrill, CD, Zegras, C, \& Ben-Akiva, M 2013, 'The Future Mobility Survey: Overview and preliminary evaluation,' in Proceedings of the Eastern Asia Society for Transportation Studies, Vol. 9.

Pierce, B, Casas, J, \& Giaimo, G 2003, 'Estimating trip rate under-reporting: Preliminary results from the Ohio travel survey,' 82nd Annual Meeting of the Transportation Research Board, Washington, D. C.

Raveau, S, Daziano, RA, Yáñez, MF, Bolduc, D, \& Ortúzar, JD 2010, 'Sequential and simultaneous estimation of hybrid discrete choice models: Some new findings,' Transportation Research Record: Journal of the Transportation Research Board, no. 2156, Transportation Research Board of the National Academies, Washington, D.C., 2010, pp. 131-139.

Reddy, S, Mun, M, Burke, J, Estrin, D, Hansen, M, \& Srivastava, M 2010, 'Using mobile phones to determine transportation modes,' ACM Transactions on Sensor Networks (TOSN), vol. 6, no. 2, pp. 13:1-13:27.

Shankari, K, Yin, M, Shanmugam, S, Culler, D, \& Katz, R (2014), 'E-Mission: Automated transportation emission calculation using smart phones,' Tech. rep. UCB/EECS-2014-140, EECS Department, University of California, Berkeley, viewed 24 April 2015, http://www.eecs.berkeley.edu/Pubs/TechRpts/2014/EECS-2014-140.html. 
Shen, L \& Stopher, PR 2014, 'Review of GPS travel survey and GPS data-processing methods,' Transport Reviews, vol. 34 , no. 3 , pp. $316-334$.

Stenneth, L, Wolfson, O, Yu, PS, \& Xu, B 2011, 'Transportation mode detection using mobile phones and GIS information,' in Proceedings of the 19th ACM SIGSPATIAL International Conference on Advances in Geographic Information Systems.

Stopher, P, FitzGerald, C, \& Zhang, J 2008, 'Search for a global positioning system device to measure person travel,' Transportation Research Part C: Emerging Technologies, vol. 16, no. 3, pp. 350-369.

Stopher, P, Wargelin, L, Minser, J, Tierney, K, Rhindress, M, \& O’Connor, S. 2012, 'GPS-based household interview survey for the Cincinnati, Ohio region,' Cincinnati, Ohio: Abt SRBI, Incorporated.

Williams, HCWL \& Ortúzar, JD 1982, 'Behavioural theories of dispersion and the misspecification of travel demand models,' Transportation Research Part B: Methodological, vol. 16, no. 3, pp. 167-219.

Wolf, J, Guensler, R, \& Bachman, W 2001, 'Elimination of the travel diary: Experiment to derive trip purpose from global positioning system travel data,' Transportation Research Record: Journal of the Transportation Research Board, vol. 1768, no. 1, pp. 125-134.

Wolf, J, Bachman, W, Oliveira, MS, Auld, J, Mohammadian, AK, \& Vovsha, P 2014, 'Applying GPS data to understand travel behavior,' National Cooperative Highway Research Program (NCHRP) Report 775.

Zheng, Y, Chen, Y, Li, Q, Xie, X, \& Ma, WY 2010, 'Understanding transportation modes based on GPS data for web applications,' ACM Transactions on the Web (TWEB), vol. 4, no. 1, pp. 1:1-1:36.

Zmud, J \& Wolf, J 2003, 'Identifying the correlates of trip misreporting - Results from the California statewide household travel survey GPS study,' in Proceedings of the 10th International Conference on Travel Behaviour Research, Lucerne, Switzerland.

Zmud, J, Lee-Gosselin, M, Carrasco, JA, \& Munizaga, M (Eds.) 2013, 'Transport survey methods: Best practice for decision making,' Emerald Group Publishing. 
Figure 1: Example confusion matrix, adapted from NCHRP Report 775 (Wolf et al., 2014), for a neural network travel mode inference algorithm

\begin{tabular}{|c|c|c|c|c|c|}
\hline & \multicolumn{4}{|c|}{ Inferred } \\
\hline & & Walk & Bike & $\mathrm{Car}$ & Transit \\
\hline \multirow{4}{*}{ 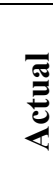 } & Walk & 0.94 & 0.00 & 0.06 & 0.00 \\
\hline & Bike & 0.00 & 0.94 & 0.06 & 0.00 \\
\hline & Car & 0.00 & 0.00 & 0.55 & 0.45 \\
\hline & Transit & 0.03 & 0.06 & 0.03 & 0.88 \\
\hline
\end{tabular}


Figure 2: General form of the confusion matrix used in experiments 1 and 2

\begin{tabular}{|c|c|c|c|c|c|}
\hline & \multicolumn{4}{|c|}{ Inferred } \\
\hline & & Walk & Bike & Car & Transit \\
\hline \multirow{4}{*}{ 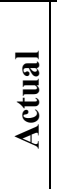 } & Walk & $\alpha$ & $\gamma$ & $\gamma$ & $\gamma$ \\
\hline & Bike & $\gamma$ & $\alpha$ & $\gamma$ & $\gamma$ \\
\hline & Car & $\gamma$ & $\gamma$ & $\alpha$ & $\gamma$ \\
\hline & Transit & $\gamma$ & $\gamma$ & $\gamma$ & $\alpha$ \\
\hline
\end{tabular}


Figure 3: A boxplot showing the median, 25th and 75th percentile, and minimum and maximum value of travel time $(\$ / \mathrm{hr})$ for experiment 1 as estimated using the unweighted likelihood function
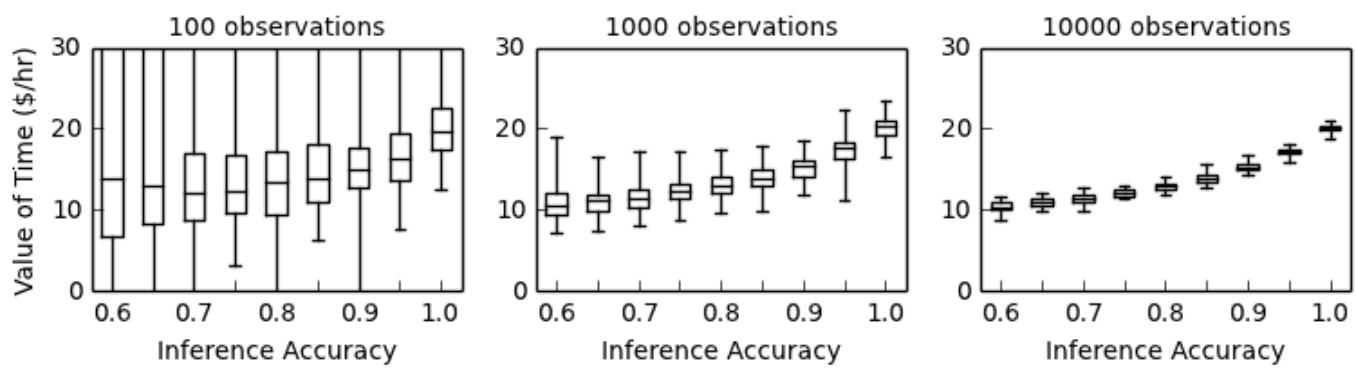
Figure 4: A boxplot showing the median, 25th and 75th percentile, and minimum and maximum value of travel time $(\$ / \mathrm{hr})$ for experiment 1 as estimated using the weighted likelihood function
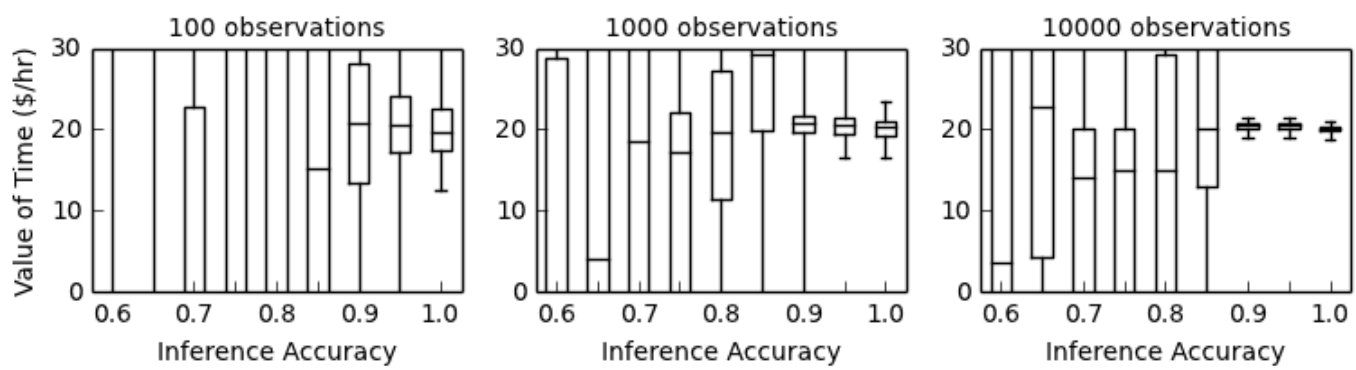
Figure 5: A boxplot showing the median, 25th and 75th percentile, and minimum and maximum value of in-vehicle travel time $(\$ / \mathrm{hr})$, value of walking time $(\$ / \mathrm{hr})$, value of biking time $(\$ / \mathrm{hr})$ and value of waiting time $(\$ / \mathrm{hr})$ for experiment 2 , as estimated using the unweighted likelihood function
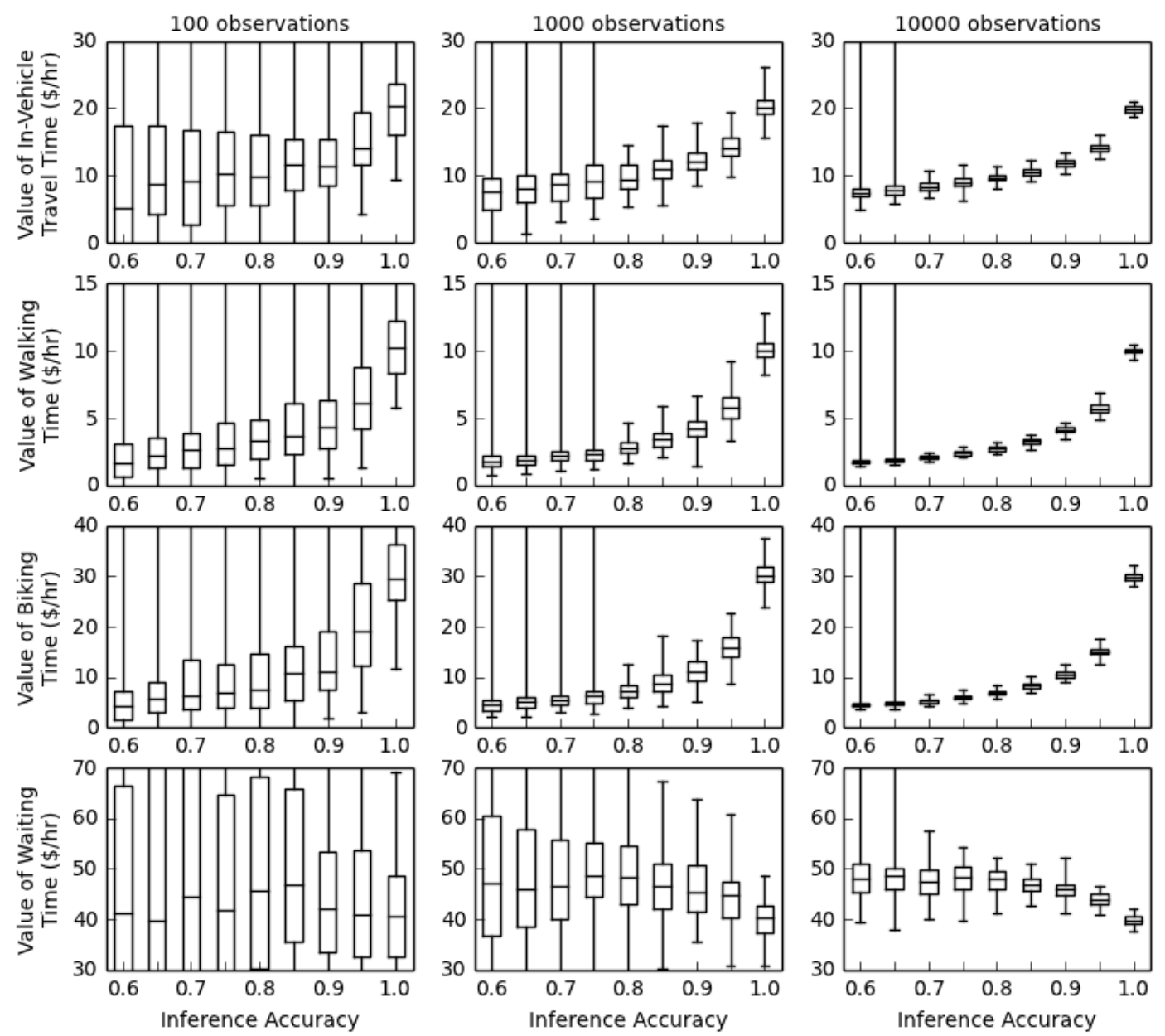
Figure 6: A boxplot showing the median, 25th and 75th percentile, and minimum and maximum value of in-vehicle travel time $(\$ / \mathrm{hr})$, value of walking time $(\$ / \mathrm{hr})$, value of biking time $(\$ / \mathrm{hr})$ and value of waiting time $(\$ / \mathrm{hr})$ for experiment 2 , as estimated using the weighted likelihood function
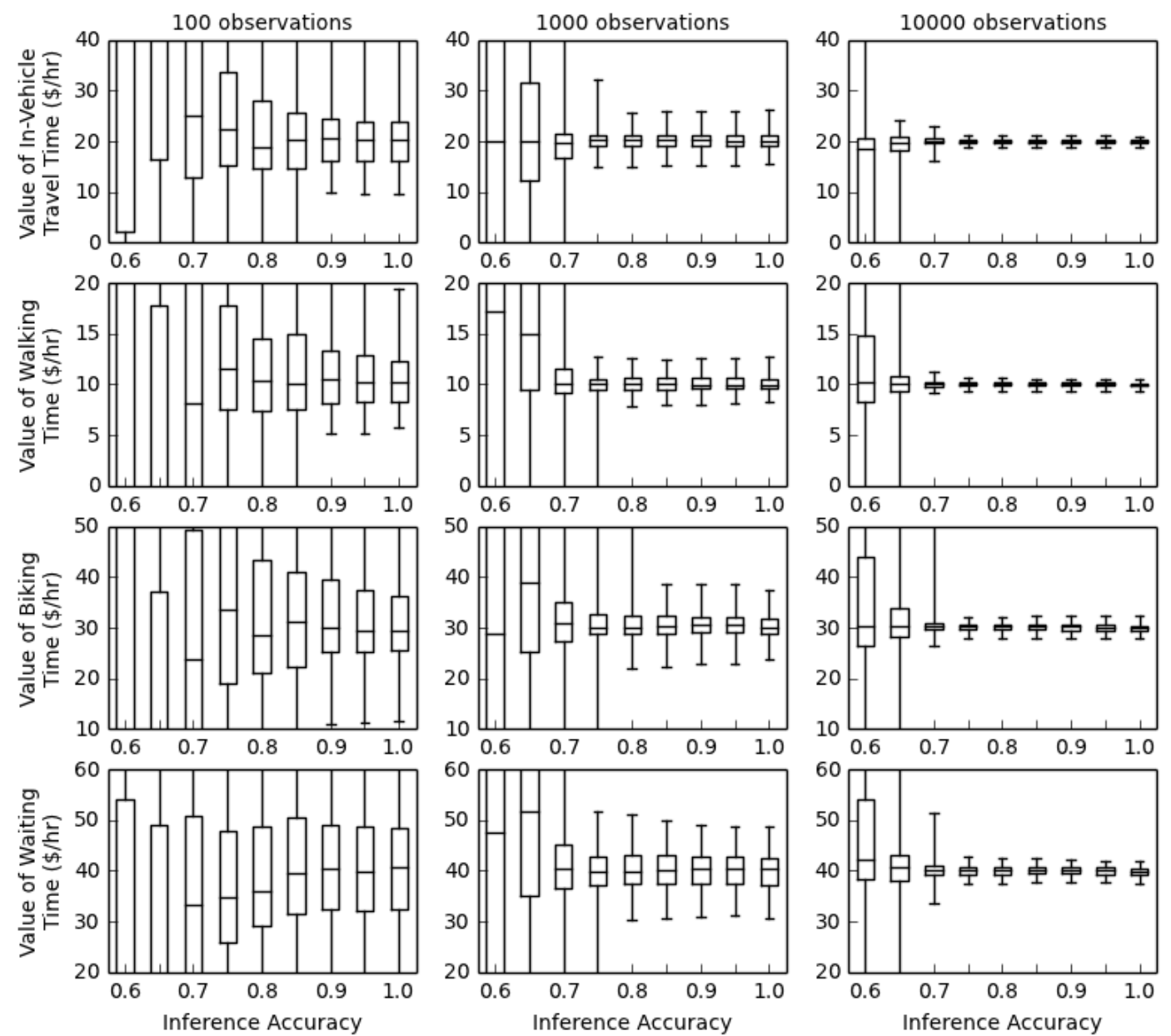
Figure 7: General form of the confusion matrix used in experiment 3

\begin{tabular}{|c|c|c|c|c|c|}
\hline & \multicolumn{4}{|c|}{ Inferred } \\
\hline & & Walk & Bike & Car & Transit \\
\hline \multirow{4}{*}{ 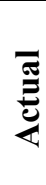 } & Walk & $\gamma$ & $\rho$ & $2 \rho$ & $\rho$ \\
\hline & Bike & $\rho$ & $\gamma$ & $2 \rho$ & $\rho$ \\
\hline & Car & $\sigma$ & $\sigma$ & $\delta$ & $8 \sigma$ \\
\hline & Transit & $\rho$ & $2 \rho$ & $\rho$ & $\gamma$ \\
\hline
\end{tabular}


Figure 8: A boxplot showing the median, 25th and 75th percentile, and minimum and maximum value of in-vehicle travel time $(\$ / \mathrm{hr})$, value of walking time $(\$ / \mathrm{hr})$, value of biking time $(\$ / \mathrm{hr})$ and value of waiting time $(\$ / \mathrm{hr})$ for experiment 3, as estimated using the unweighted likelihood function
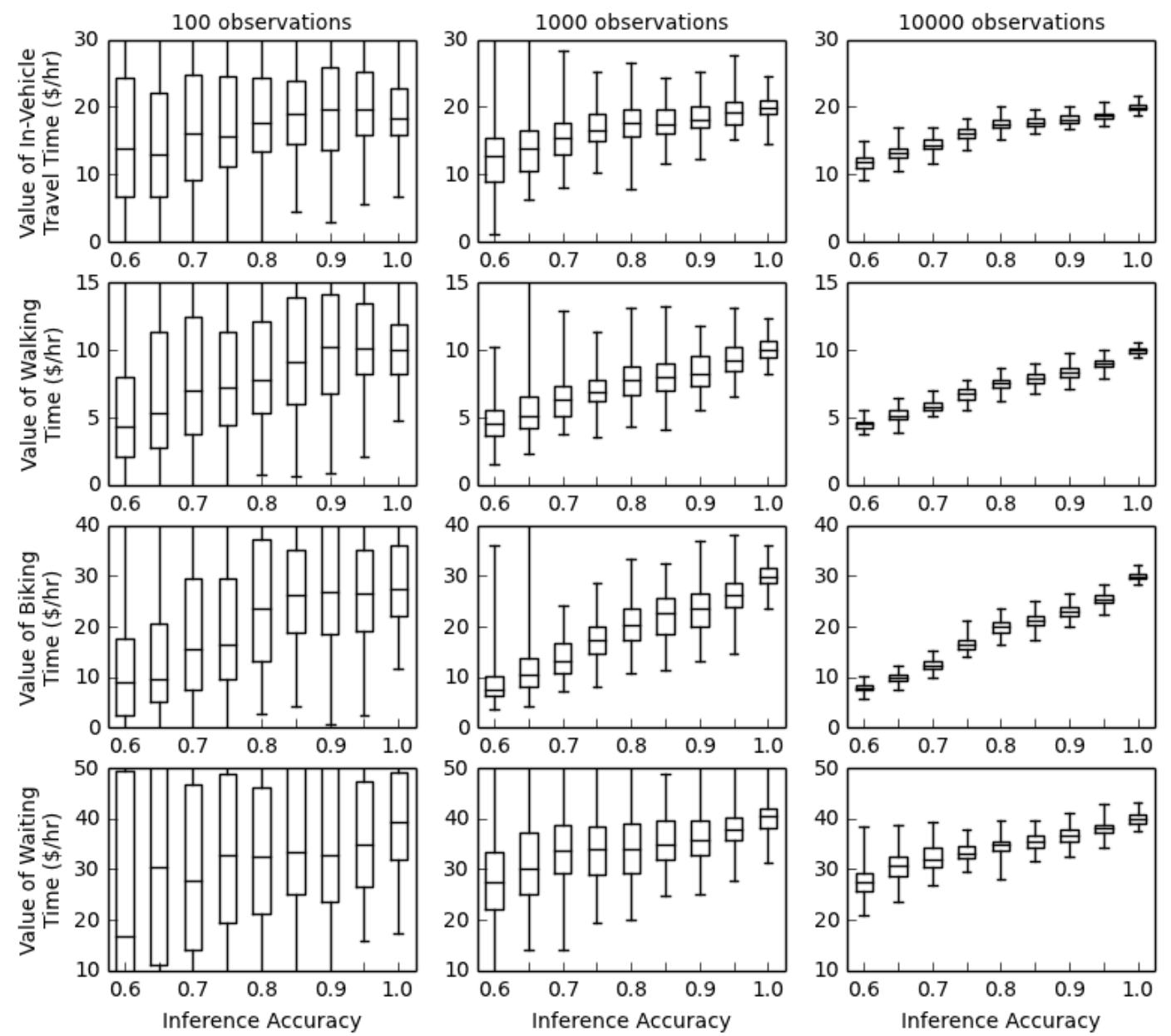
Figure 9: A boxplot showing the median, 25th and 75th percentile, and minimum and maximum value of in-vehicle travel time $(\$ / \mathrm{hr})$, value of walking time $(\$ / \mathrm{hr})$, value of biking time $(\$ / \mathrm{hr})$ and value of waiting time $(\$ / \mathrm{hr})$ for experiment 3, as estimated using the weighted likelihood function
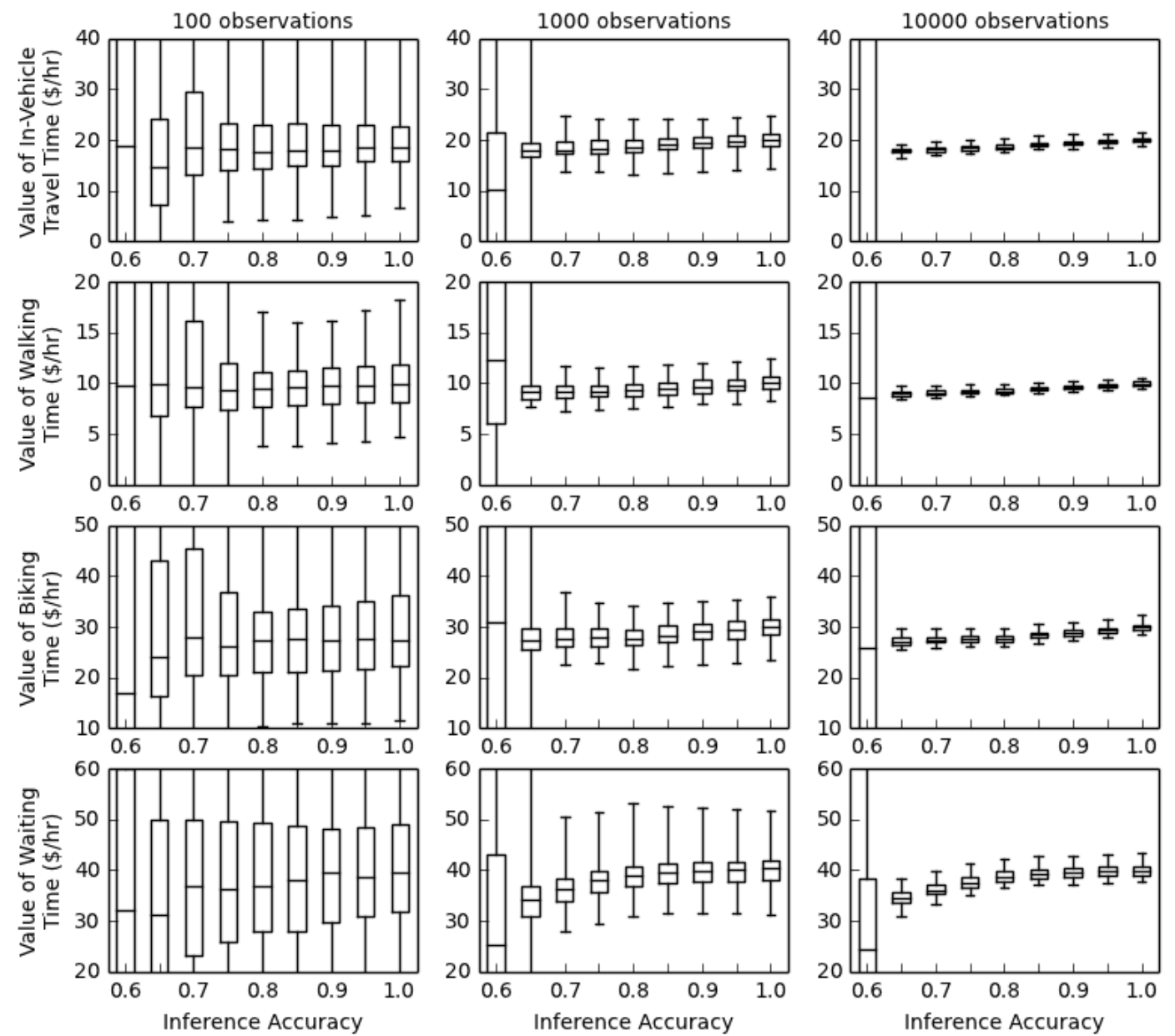
Figure 10: A plot comparing the value of time $(\$ / \mathrm{hr})$ as estimated by maximizing the unweighted and weighted likelihood functions given by equations (11) and (12), respectively, as a function of the average accuracy of the inference algorithm

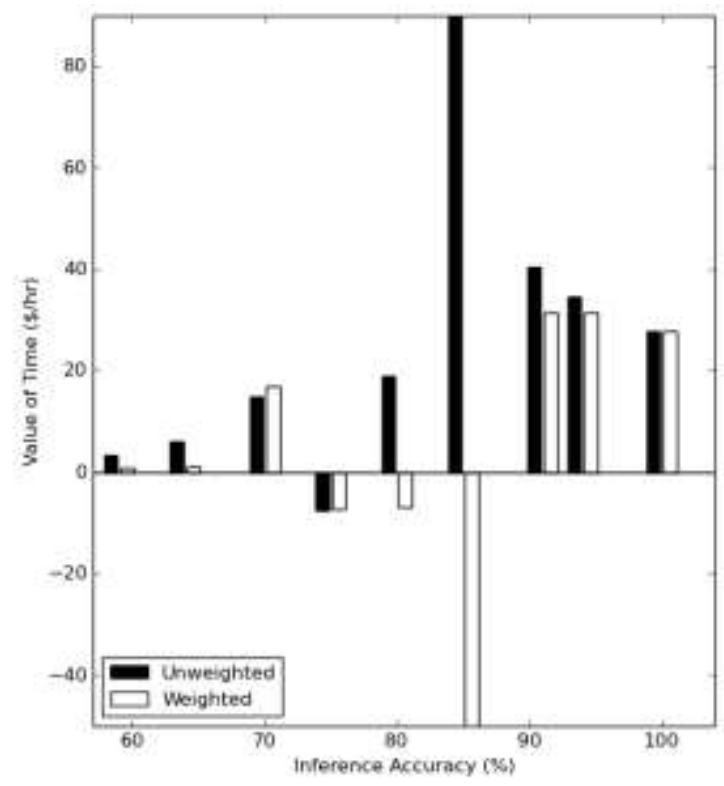


Figure 11: A bar and line plot where the bar plot represents the value of time ( $\$ / \mathrm{hr})$ estimated by maximizing the weighted likelihood function given by equation (5) and the inferred data is supplemented with ground truth, and the line plot represents the percentage of trips for which the accuracy of the inference algorithm is below $90 \%$ and ground truth is used, plotted as a function of the average accuracy of the inference algorithm

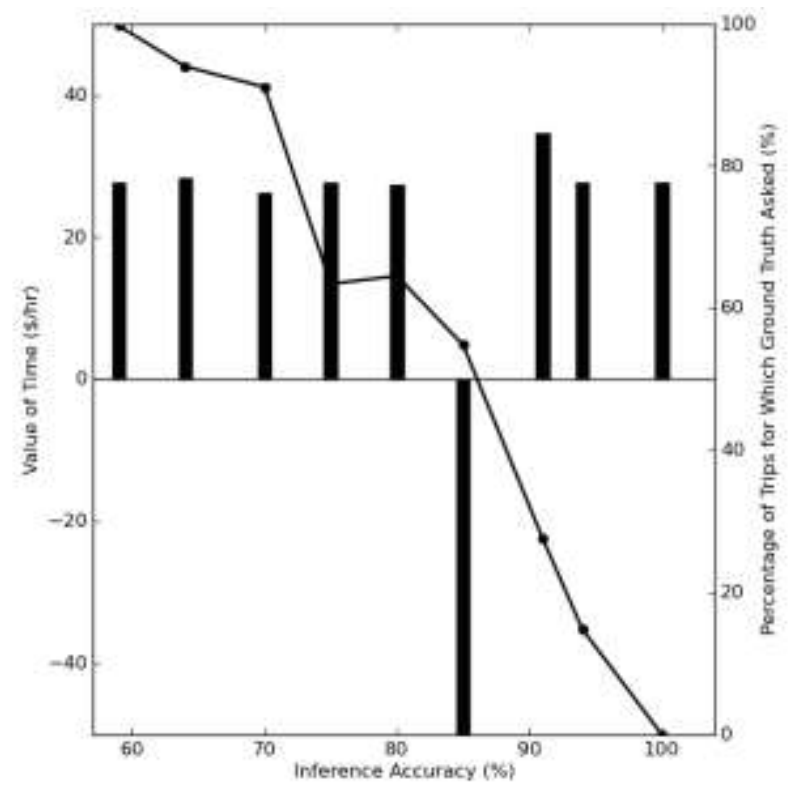


Table 1: Distributions for travel mode level-of-service attributes for experiment 1

\begin{tabular}{lllr}
\hline Variable & Notation & Units & Distribution \\
\hline Travel time - Walk & $\mathrm{tt}_{\text {walk }}$ & Minutes & $\mathrm{U}\left(1.5 \mathrm{tt}_{\mathrm{car}}, 2.5 \mathrm{tt}_{\mathrm{car}}\right)$ \\
Travel time - Bike & $\mathrm{tt}_{\text {bike }}$ & Minutes & $\mathrm{U}\left(\mathrm{tt}_{\mathrm{car}}, 1.5 \mathrm{tt}_{\mathrm{car}}\right)$ \\
Travel time - Car & $\mathrm{tt}_{\text {car }}$ & Minutes & $\mathrm{U}(10,60)$ \\
Travel time - Transit & $\mathrm{tt}_{\text {transit }}$ & Minutes & $\mathrm{U}\left(\mathrm{tt}_{\mathrm{car}}, 2.5 \mathrm{tt}_{\mathrm{car}}\right)$ \\
Speed - Car & $\mathrm{v}_{\text {car }}$ & Mph & $\mathrm{LN}(2.05,0.63)$ \\
Distance - Car & $\mathrm{s}_{\text {car }}$ & Miles & $\mathrm{v}_{\text {car }} \mathrm{tt}_{\mathrm{car}} / 60$ \\
Cost - Walk & cost $_{\text {walk }}$ & $\$$ & 0 \\
Cost - Bike & cost $_{\text {bike }}$ & $\$$ & 0 \\
Cost - Car & cost $_{\text {car }}$ & $\$$ & $\mathrm{U}(0,5)+0.6 \mathrm{~s}_{\mathrm{car}}$ \\
Cost - Transit & cost $_{\text {transit }}$ & $\$$ & $\mathrm{U}(0,4)$ \\
\hline
\end{tabular}

$\mathrm{U}(\mathrm{a}, \mathrm{b})$ denotes a uniform distribution over the range $(\mathrm{a}, \mathrm{b})$

$\mathrm{LN}(\mathrm{a}, \mathrm{b})$ denotes a lognormal distribution with location $\mathrm{a}$ and scale $\mathrm{b}$ 
Table 2: Parameter values for experiment 1

\begin{tabular}{lllr}
\hline Parameter & Notation & Units & Value \\
\hline Constant - Walk & $\mathrm{ASC}_{\text {walk }}$ & Utils & 0.00 \\
Constant - Bike & $\mathrm{ASC}_{\text {bike }}$ & Utils & -2.50 \\
Constant - Car & $\mathrm{ASC}_{\text {car }}$ & Utils & 1.00 \\
Constant - Transit & $\mathrm{ASC}_{\text {transit }}$ & Utils & -0.50 \\
Travel time & $\beta_{\mathrm{tt}}$ & Utils/minute & -0.20 \\
Travel cost & $\beta_{\text {cost }}$ & Utils $/ \$$ & -0.60 \\
\hline
\end{tabular}


Table 3: Distributions for travel mode level-of-service attributes for experiments 2 and 3

\begin{tabular}{|c|c|c|c|}
\hline Variable & Notation & Units & Distribution \\
\hline In-vehicle travel time - Walk & ivtt $_{\text {walk }}$ & Minutes & 0 \\
\hline In-vehicle travel time - Bike & ivtt $_{\text {bike }}$ & Minutes & 0 \\
\hline In-vehicle travel time - Car & $\mathrm{ivtt}_{\mathrm{car}}$ & Minutes & $\mathrm{U}(10,50)$ \\
\hline In-vehicle travel time - Transit & ivtt $_{\text {transit }}$ & Minutes & $\mathrm{U}\left(0.8\right.$ ivtt $_{\mathrm{car}}, 1.5$ ivtt $\left._{\mathrm{car}}\right)$ \\
\hline Speed - Walk & $\mathrm{v}_{\text {walk }}$ & Mph & $\operatorname{LN}(0.28,0.43)$ \\
\hline Speed - Bike & $v_{\text {bike }}$ & Mph & $\operatorname{LN}(1.38,0.38)$ \\
\hline Speed - Car & $\mathrm{v}_{\text {car }}$ & $\mathrm{Mph}$ & $\operatorname{LN}(2.05,0.63)$ \\
\hline Distance - Car & $\mathrm{s}_{\mathrm{car}}$ & Miles & $\mathrm{v}_{\mathrm{car}} \mathrm{ivtt}_{\mathrm{car}} / 60$ \\
\hline Walking time - Walk & wktt $_{\text {walk }}$ & Minutes & $60 \mathrm{~s}_{\mathrm{car}} / \mathrm{v}_{\mathrm{walk}}$ \\
\hline Walking time - Bike & wktt $_{\text {bike }}$ & Minutes & 0 \\
\hline Walking time - Car & wktt $_{\text {car }}$ & Minutes & $\mathrm{U}(0,10)$ \\
\hline Walking time - Transit & $\mathrm{wktt}_{\text {transit }}$ & Minutes & $\mathrm{U}(0,15)$ \\
\hline Biking time - Walk & bktt $_{\text {walk }}$ & Minutes & 0 \\
\hline Biking time - Bike & bktt $_{\text {bike }}$ & Minutes & $60 \mathrm{~s}_{\mathrm{car}} / \mathrm{v}_{\mathrm{bike}}$ \\
\hline Biking time - Car & bktt $_{\mathrm{car}}$ & Minutes & 0 \\
\hline Biking time - Transit & bktt $_{\text {transit }}$ & Minutes & 0 \\
\hline Waiting time - Walk & $\mathrm{wttt}_{\text {walk }}$ & Minutes & 0 \\
\hline Waiting time - Bike & $w_{t t t}$ bike & Minutes & 0 \\
\hline Waiting time - Car & wttt $_{\mathrm{car}}$ & Minutes & 0 \\
\hline Waiting time - Transit & $w_{t t t}$ transit & Minutes & $\mathrm{U}(0,15)$ \\
\hline Cost - Walk & $\operatorname{cost}_{\text {walk }}$ & $\$$ & 0 \\
\hline Cost - Bike & $\operatorname{cost}_{\text {bike }}$ & $\$$ & 0 \\
\hline Cost - Car & $\operatorname{cost}_{\mathrm{car}}$ & $\$$ & $\mathrm{U}(0,5)+0.6 \mathrm{~s}_{\mathrm{car}}$ \\
\hline Cost - Transit & cost $_{\text {transit }}$ & $\$$ & $\mathrm{U}(0,4)$ \\
\hline
\end{tabular}

$\mathrm{U}(\mathrm{a}, \mathrm{b})$ denotes a uniform distribution over the range $(\mathrm{a}, \mathrm{b})$

$\mathrm{LN}(\mathrm{a}, \mathrm{b})$ denotes a lognormal distribution with location $\mathrm{a}$ and scale $\mathrm{b}$ 
Table 4: Parameter values for experiments 2 and 3

\begin{tabular}{lllr}
\hline Parameter & Notation & Units & Value \\
\hline Constant - Walk & $\mathrm{ASC}_{\text {walk }}$ & Utils & 0.0000 \\
Constant - Bike & $\mathrm{ASC}_{\text {bike }}$ & Utils & -0.5000 \\
Constant - Car & $\mathrm{ASC}_{\text {car }}$ & Utils & 1.0000 \\
Constant - Transit & $\mathrm{ASC}_{\text {transit }}$ & Utils & 0.5000 \\
In-vehicle travel time & $\beta_{\text {ivtt }}$ & Utils/minute & -0.1950 \\
Walking time & $\beta_{\text {wktt }}$ & Utils/minute & -0.0975 \\
Biking time & $\beta_{\text {bktt }}$ & Utils/minute & -0.2925 \\
Waiting time & $\beta_{\text {wttt }}$ & Utils/minute & -0.3900 \\
Travel cost & $\beta_{\text {cost }}$ & Utils $/ \$$ & -0.5850 \\
\hline
\end{tabular}


Table 5: Elements of the confusion matrix used in experiment 3 as a function of the average accuracy of the inference algorithm

\begin{tabular}{lrrrr}
\hline $\begin{array}{l}\text { Average } \\
\text { accuracy }(\alpha)\end{array}$ & $\boldsymbol{\gamma}$ & $\boldsymbol{\delta}$ & $\boldsymbol{\rho}$ & $\boldsymbol{\sigma}$ \\
\hline $60 \%$ & $77 \%$ & $48 \%$ & $6 \%$ & $5 \%$ \\
$65 \%$ & $84 \%$ & $52 \%$ & $4 \%$ & $5 \%$ \\
$70 \%$ & $90 \%$ & $56 \%$ & $2 \%$ & $4 \%$ \\
$75 \%$ & $97 \%$ & $60 \%$ & $1 \%$ & $4 \%$ \\
$80 \%$ & $100 \%$ & $67 \%$ & $0 \%$ & $3 \%$ \\
$85 \%$ & $100 \%$ & $75 \%$ & $0 \%$ & $3 \%$ \\
$90 \%$ & $100 \%$ & $83 \%$ & $0 \%$ & $2 \%$ \\
$95 \%$ & $100 \%$ & $92 \%$ & $0 \%$ & $1 \%$ \\
$100 \%$ & $100 \%$ & $100 \%$ & $0 \%$ & $0 \%$ \\
\hline
\end{tabular}

